\title{
Endovascular Repair for Abdominal Aortic Aneurysm Reduces Postoperative Blood Endotoxin Levels Assayed by the EAA Method Compared with Open Abdominal Surgery
}

\author{
Atsumi Ohishi, Satoshi Matsushita*, Shizuyuki Dohi, Taira Yamamoto, Hirotaka Inaba, \\ Kenji Kuwaki, Atsushi Amano \\ Department of Cardiovascular Surgery, Faculty of Medicine, Juntendo University, Tokyo, Japan \\ Email: mohishi@juntendo.ac.jp, saty-m@juntendo.ac.jp, shiz-d@juntendo.ac.jp, tyamamo@juntendo.ac.jp, \\ hinaba@juntendo.ac.jp, kkuwaki@juntendo.ac.jp, a-amano@juntendo.ac.jp
}

Received 4 May 2014; revised 4 June 2014; accepted 11 June 2014

Copyright (C) 2014 by authors and Scientific Research Publishing Inc.

This work is licensed under the Creative Commons Attribution International License (CC BY).

http://creativecommons.org/licenses/by/4.0/

c) (i) Open Access

\begin{abstract}
Introduction: We hypothesized to demonstrate whether there are significant differences in blood endotoxin (Et) levels after abdominal aortic surgery between endovascular aortic repair (EVAR) and open abdominal surgery. Methods: The patients who underwent the surgical treatment for abdominal aortic aneurysm were divided into two groups according to the procedures: open abdomen surgery (OP) and EVAR (SG). The value of Endotoxin Activity Assay (EAA) was compared between groups. Results: After surgery, Et level was significantly higher in the OP group than in the SG group on postoperative day 3 . Neutrophil count was significantly higher in the OP group immediately after treatment, but no significant difference was seen thereafter. There were no differences between the groups in other inflammatory markers. Conclusions: This study indicated that EVAR was less invasive compared to an open abdominal surgery from the standpoint of assessing postoperative endotoxin activity (EA) levels measured by EAA.
\end{abstract}

\section{Keywords}

Inflammation, Infection, Stents, Endovascular Aortic Repair

\footnotetext{
${ }^{*}$ Corresponding author.

How to cite this paper: Ohishi, A., et al. (2014) Endovascular Repair for Abdominal Aortic Aneurysm Reduces Postoperative Blood Endotoxin Levels Assayed by the EAA Method Compared with Open Abdominal Surgery. World Journal of Cardiovascular Surgery, 4, 87-94. http://dx.doi.org/10.4236/wjcs.2014.46014
} 


\section{Introduction}

Bacterial endotoxin or Endotoxin, a cellular structural component of gram negative organisms, is known to increase in the blood when perioperative sepsis or anastomotic insufficiency occurs after an intestinal surgery. This is known to be closely related to postoperative prognosis. However, elevation of plasma endotoxin levels is sometimes reported even when no remarkable infection has been detected [1]. It is suspected that this phenomenon is a result of the translocation of endotoxin from the intestines that are handled during surgery. Interestingly, endotoxemia is also reported in patients who have undergone open heart surgeries that do not touch the intestines [2], and this is also relevant to prolonged hospital stays and postoperative complications [3]. Although this may be explained as translocation caused by the transient ischemic condition of the intestines due to cardiopulmonary bypass during surgery, the mechanism is not revealed in detail [4]. Some reports speculate that endotoxin is relevant to the release of other inflammatory markers such as CRP or IL-6 [5]. Therefore, it has been suggested that blood endotoxin is not only an indicator for gram-negative bacterial infections but also a useful biomarker for postoperative outcomes.

Previously, open abdominal surgery has been performed as a general approach for the treatment of abdominal aortic aneurysm. However, recent development in endovascular aneurysm repair (EVAR) has rapidly expanded its clinical use, and its advantages such as minimal invasiveness, lower mortality rates, decrease in average ICU stay [6] and hospital stay [7]-[9], and less perioperative complications [10] have been reported. Therefore, we hypothesized that postoperative blood EA levels were lower after EVAR than after an open abdominal surgery. In this study, whole blood EA levels were measured using the new Endotoxin Activity Assay (EAA) which has been reported to allow accurate and precise quantification of trace amounts of endotoxin in vitro studies. Using this method, a more practical measurement could be provided by targeting active endotoxin in sepsis [11]-[13]. As it implicates that the correlation between blood endotoxin and the severity of inflammation, quantitative measurements using the EAA method may be an attractive candidate for a new biomarker of inflammation.

The purpose of this study is to analyze the minimal invasiveness of endovascular aneurysm repair from the viewpoint of blood endotoxin, to examine how this relates to the patient's postoperative course, and to reveal the clinical significance of quantitative measurement of endotoxin by the EAA method.

\section{Methods}

\subsection{Human Specimen Handling}

Patients with abdominal aortic aneurysm who underwent either open abdominal surgery or endovascular aneurysm repair at Juntendo University Hospital were studied. Peripheral blood sample was used, and it was handled according to the Declaration of Helsinki. The study was approved by the Human Ethics Committee of Juntendo University. A written consent was signed by each patient prior to the surgery.

\subsection{Human Subjects}

Patients treated for abdominal aortic aneurysm from April to December 2012 were divided into two groups. The first group underwent open abdominal surgery (OP), and the other group underwent endovascular aneurysm repair (SG). Cases were omitted if the patient received preoperative steroid treatment, the surgery combined multiple procedures such as open heart surgery, or the patient had inflammatory diseases such as vasculitis. Preoperative peripheral blood was taken to measure the baseline levels of endotoxin (Et), body temperature, white blood cell count (WBC), CRP, tumor necrosis factor $\alpha$ (TNF- $\alpha$ ), and interleukin 6 (IL-6). Among them, WBC and CRP were measured in central laboratory of Juntendo University, and TNF- $\alpha$, IL-6 were measured by outsourcing laboratory (SRL Inc, Tokyo). These values were also measured postoperatively until postoperative day 5 to calculate the rate of change. Patient information such as age, gender, BMI, medication, complications, previous surgical history including procedure, device used, operative time, and postoperative course were recorded and compared between the two groups.

\subsection{Endotoxin Measurement}

Spectral's Endotoxin Activity Assay (EAA, Toray Medical Co. Ltd., Tokyo) was used for the measurement of whole blood endotoxin. The presence of endotoxin or lipopolysaccharide (LPS), an outer membrane component 
of gram-negative bacteria, can result in immune complex formation in the presence of a specific anti-LPS antibody to yield complement fixation. Then, the opsonized zymosan primes neutrophils in the blood to trigger their oxidative burst.

A chemilumine scence assay was performed according to the manufacture's instruction. Actual measurements were made of light emissions over a total period of $15 \mathrm{~min}$ in a tubeluminometer (Auto Lumat LB953, E.G. \& G, Berthold, Wildbat, Germany) and quantitative analysis was completed within 2 hours of sample collection. The relative light units (RLU) measured by the instrument was converted by calculation into an endotoxin activity (EA) value.

\subsection{Statistical Analysis}

Each measurement was recorded as average \pm standard error. Comparison between the 2 groups was made using Statcel 3 software (OMS publishing Inc, Tokyo). Statistical significance was determined using the Student's $t$-test, and $\mathrm{P}<0.05$ was considered significantly different. The comparison of the peak level of Et with preoperative value in each patient was analyzed by the paired $t$-test.

\section{Results}

\subsection{Patient Background}

During the study period, 17 cases of abdominal aortic aneurysms were treated. Open abdominal surgeries (OP group) were performed in 9 of these cases, and endovascular aneurysm repairs (SG group) were performed in 8 cases. Patients with preoperative endotoxin (Et) above the low level cutoff value (0.4) were excluded from the study. The study was performed in 14 remaining cases, 7 cases in each group, which met none of the other exclusion criteria.

Preoperative patient background is shown in Table 1. The OP group consisted of all male patients, and the SG group consisted of 4 male and 3 female patients. The average age of patients were $70.6 \pm 8.5$ (OP) and 71.1 \pm 7.3 (SG) and did not show significant difference. There were no significant differences in preoperative BMI, diabetes, hypertension, hyperlipidemia and smoking history. Serum creatinine (Cre) were significantly higher in the OP group than in the SG group (1.63 \pm 0.89 vs $0.73 \pm 0.12, \mathrm{P}=0.02)$. No significant difference was seen in preoperative endotoxin (Et) between the groups $(0.17 \pm 0.07$ vs $0.19 \pm 0.06, P=0.47)$. There were no significant differences in other variables such as body temperature, WBC, CRP, TNF- $\alpha$, and IL-6 as illustrated in Table 2. The OP group consisted of 5 patients with abdominal aortic aneurysm and 2 with arteriosclerosis obliterans, and all patients in the SG group had abdominal aortic aneurysm. Operative time and postoperative hospital stay were significantly longer in the OP group, and there were no significant difference in blood transfusion or ICU stay (Table 3).

Table 1. Baseline characteristics.

\begin{tabular}{cccc}
\hline & OP $(\mathrm{n}=7)$ & SG $(\mathrm{n}=7)$ & P Value \\
\hline Age, year & $70.6 \pm 8.5$ & $71.1 \pm 7.3$ & 0.89 \\
Female (\%) & $0(0)$ & $3(42.9)$ & 0.12 \\
BMI, kg/m² & $23.6 \pm 2.7$ & $25.7 \pm 4.5$ & 0.29 \\
DM (\%) & $1(14.2)$ & $1(14.2)$ & 1.00 \\
HT (\%) & $6(85.7)$ & $4(57.1)$ & 0.24 \\
HL (\%) & $5(83.3)$ & $4(57.1)$ & 0.58 \\
Smoking history (\%) & $6(85.7)$ & $4(57.1)$ & 0.24 \\
Creatinine, mg/dL & $1.63 \pm 0.89$ & $0.73 \pm 0.12$ & 0.02
\end{tabular}

*BMI: body mass index, DM: diabetes mellitus, HT: hypertension, HL: hyperlipidemia, creatinine: serum creatinine. 
Table 2. Preoperative values.

\begin{tabular}{cccc}
\hline & OP $(\mathrm{n}=7)$ & SG $(\mathrm{n}=7)$ & P Value \\
\hline Et & $0.17 \pm 0.07$ & $0.19 \pm 0.06$ & 0.47 \\
WBC, $\times 10^{3} / \mu \mathrm{L}$ & $6.4 \pm 1.2$ & $5.1 \pm 1.5$ & 0.29 \\
Neutrophil, $\times 10^{3} / \mu \mathrm{L}$ & $4.1 \pm 0.8$ & $3.1 \pm 0.9$ & 0.04 \\
CRP, mg/dL & $0.36 \pm 0.45$ & $0.10 \pm 0.06$ & 0.16 \\
TNF- $\alpha, \mathrm{pg} / \mathrm{mL}$ & $1.91 \pm 1.27$ & $1.39 \pm 0.36$ & 0.31 \\
IL-6, pg/mL & $3.37 \pm 2.99$ & $2.71 \pm 1.33$ & 0.60 \\
BT, Celsius degree & $36.4 \pm 0.4$ & $36.3 \pm 0.5$ & 0.64
\end{tabular}

*Et: endotoxin, BT: body temperature, WBC: white blood cells, CRP: C-reactive protein TNF- $\alpha$ : tumor necrosis factor-alpha, IL-6: interleukin-6.

Table 3. Surgical indices.

\begin{tabular}{cccc}
\hline & OP $(\mathrm{n}=7)$ & SG $(\mathrm{n}=7)$ & P Value \\
\hline Operation time, minutes & $327.4 \pm 102.7$ & $167.7 \pm 100.7$ & 0.01 \\
Blood transfusion, \% & 42.9 & 14.2 & 0.12 \\
Aortic Clamp time, minutes & $119.4 \pm 35.6$ & N/A & N/A \\
ICU stay, day & $1.4 \pm 0.5$ & $1.4 \pm 1.1$ & 1.00 \\
After operation & $13.9 \pm 4.5$ & $8.0 \pm 6.4$ & 0.07 \\
\hline
\end{tabular}

\subsection{Endotoxin}

Figure 1 shows the sequential changes in EA levels. Although no significant difference between the 2 groups was seen on the day of surgery to 1 day after surgery, the value increased significantly in the OP group 3 days after surgery $(0.38 \pm 0.06$ vs $0.26 \pm 0.08$, OP vs SG, $\mathrm{P}=0.01)$. This value in the OP group decreased on day 5 , and no significant difference was seen compared with the SG group.

The peak level of EA in each patient was compared with the preoperative baseline (Figure 2). The EA values were increased in all patients of the OP group. In contrast, while the peak Et value in the SG group showed an increase compared to the baseline in most cases, some of them were unchanged or slightly decreased. The mean value increased significantly after surgery in both $O P(P=0.002)$ and SG groups $(P=0.035)$, and the slope was steeper in the OS group than the SG group.

\subsection{Other Inflammatory Markers}

WBC, neutrophil count, CRP, TNF- $\alpha$, IL-6, and body temperature were measured as inflammatory markers (Figure 3). WBC and neutrophil count were both significantly higher in the OP group immediately after the surgery $(\mathrm{P}<0.01)$, but did not show significant differences subsequently. No significant difference in serum CRP levels between the 2 groups was observed during the study. The OP group showed higher levels of serum TNF- $\alpha$ compared to those in the SG group throughout the study, but the difference was not significant. Serum IL-6 reached peak level on postoperative day 1, and then subsequently decreased in both groups. The level was slightly higher in the OP group on postoperative day 3 , but the difference was not significant $(\mathrm{P}=0.86)$. Change in body temperature was slightly higher in the SG group and prolonged fever occurred, but the increase was not significant $(\mathrm{P}<0.09)$.

\section{Discussion}

In this study, perioperative whole blood endotoxin levels were measured sequentially using the EAA method. There have been many reports indicating correlations between blood EA level and clinical outcomes [2] [5] [12] 


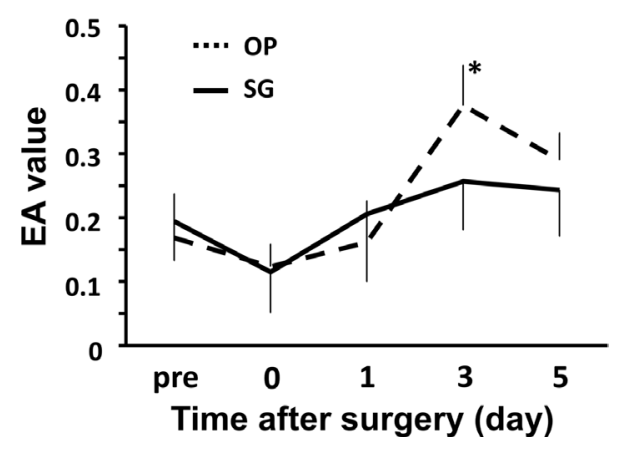

Figure 1. Endotoxin activity. The mean endotoxin activity (EA) value in OP and SG groups are shown as break line and solid line, respectively. The preoperative values in two groups were not statistically different from one another. The values decreased on the day of surgery in both groups. On the third day after surgery, the value was significantly higher in OP group compared to that of SG $(P=0.011)$.

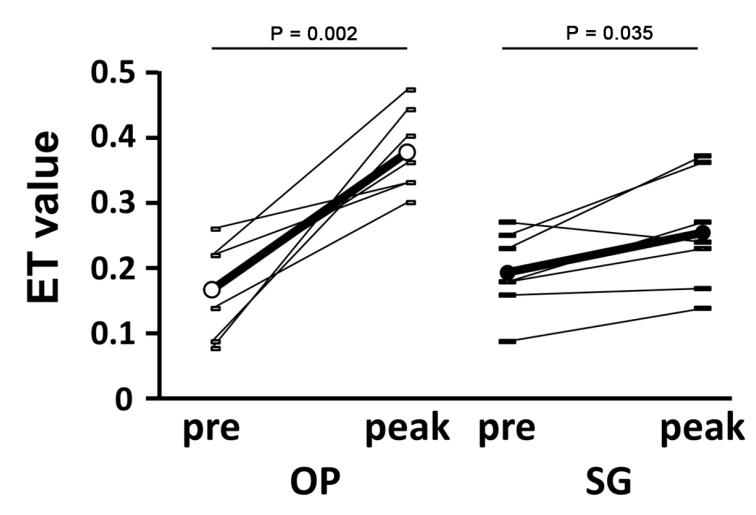

Figure 2. Individual changes in endotoxin activity. The change in endotoxin activity (EA) values in each patient from preoperative baseline to the peak postoperative value is shown. The change in the mean values of the 2 groups (thick line) represented a significantly larger increase in the OP group $(\mathrm{P}=0.002)$ compared to that in the $\mathrm{SG}$ group $(\mathrm{P}=0.035)$.

[14]-[17]. However few study has compared the endotoxin between open surgery and endovascular treatment for abdominal aortic aneurysm [18]-[22]. The primary finding of this study is that blood endotoxin was significantly increased after open surgery compared to endovascular treatment on the third day after surgery, even though this study has found no evidence of infection-related complications. The secondary finding is that there was no correlation between the levels of EA and other inflammatory markers, implying that endotoxin is an independent marker for systemic inflammation.

\subsection{Clinical Significance of Postoperative Endotoxin Elevation}

Previous reports indicate that high levels of postoperative endotoxin lead to prolonged ICU and hospital stay. However, there are few reports which track the change in post operative EA levels for up to five days after treatment. Klein et al. performed the sequential determinations of perioperative EA levels in patients who underwent open heart surgeries with cardiopulmonary bypass by using the EAA method, and concluded that high levels of endotoxin increase the risk of infection [3]. However, the measurements were only performed immediately after aortic clamping and on postoperative day 1 (within 24 hours). Previous studies demonstrated that the endotoxin level was the highest during cardiopulmonary bypass, and then it decreased with time [5] [23]. 
(a)

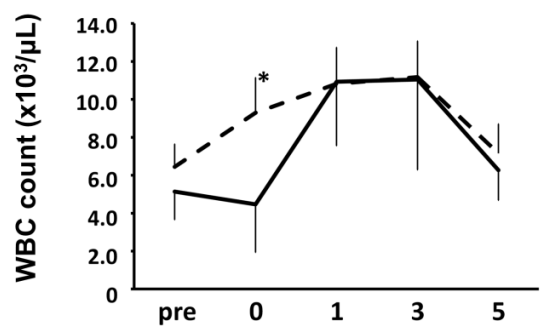

(c)

(e)
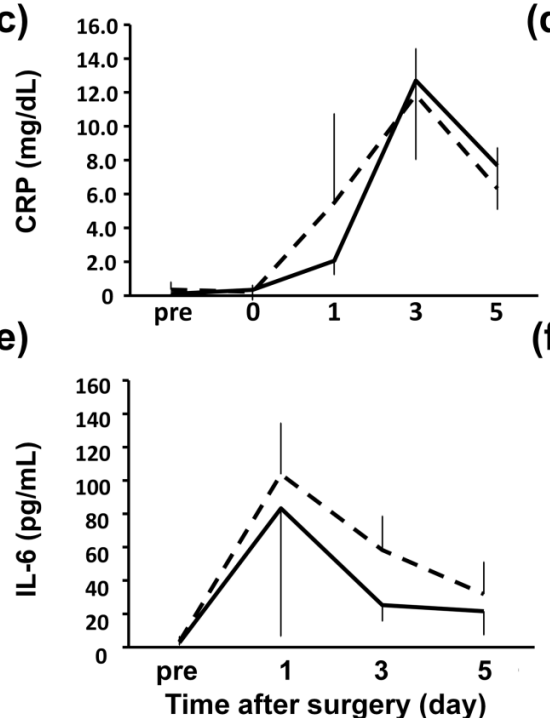

(b)

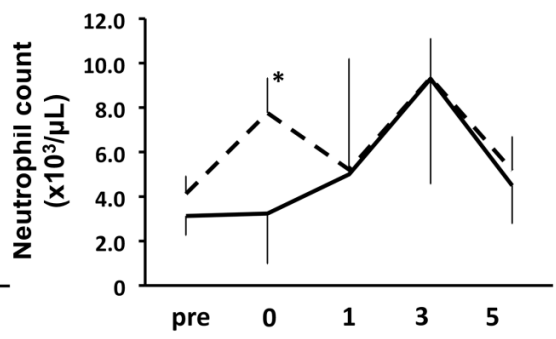

(d)
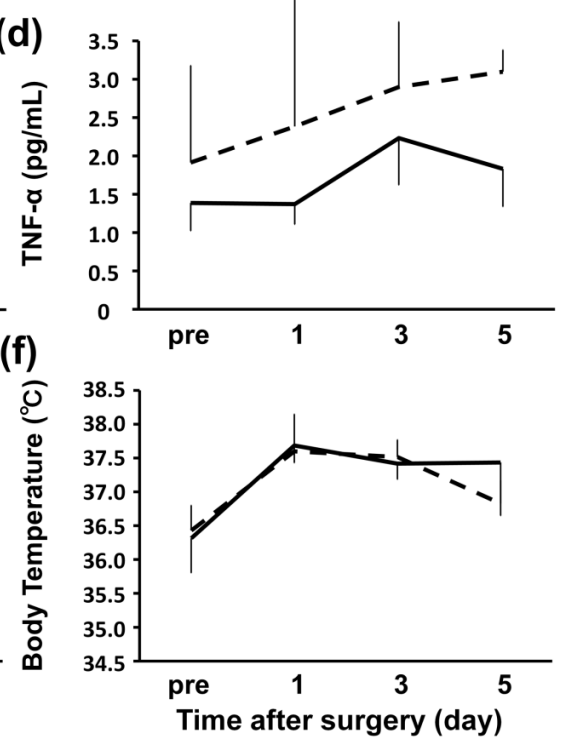

Figure 3. Changes in inflammation markers. Time course changes of other inflammation markers. Although WBC and neutrophil were significantly higher only on the day of surgery in OP, as for other markers, a statistical analysis did not show any significant differences between the groups during the study period $(\mathrm{P}<0.05)$.

Contrastively, in our current study, endotoxin levels did not present significant increase until the first day of surgery in either group, but all cases in the OP group showed an increase until the third day of surgery. The endotoxin levels in three of these cases exceeded 0.4, the low level cutoff set by the MEDIC Study [12].

Intestinal handling is thought to be the reason behind the elevation of postoperative EA levels in the OP group. All cases in the OP group involved infrarenal aortic clamping, and there were no remarkable events in the intestinal blood supply from the celiac artery and the superior mesenteric artery. As in other general operative procedures, surgical view was kept clear using devices such as a retractor. It has been previously suggested that intestinal translocation could increase blood endotoxin [23], and it is suspected that intestinal retraction causes this increase on postoperative day 3 when the diet is generally started in patients who underwent open abdominal surgery. In addition, the traditional Limulus test was performed with Limulus Amebocyte Lysate (LAL) assay kit (Wako pure chemical industrial, Tokyo, Japan) in serum blood samples by outsourcing laboratory (SRL Inc.), which showed the highest level of postoperative endotoxin in the OP group, but the levels did not exceed the reference value $(1.0 \mathrm{pg} / \mathrm{mL})$ at any point after surgery. The tendency of postoperative EA levels to increase a few days after surgery became evident from our analysis, but the mechanism behind it is still unclear and requires further investigation.

Meanwhile, there were 3 cases with preoperative endotoxin levels of 0.4 or above which were excluded from the study. Of these cases, preoperative pneumonia and pulmonary metastasis were suspected in one case, and the other two did not display any evidence of infection though Buerger's disease was present in one of them. Taking into account the MEDIC Study which reports endotoxin levels of $0.4-0.6$ were seen in approximately $7 \%$ of healthy volunteers, there could be a problem of nonspecific elevations in three excluded cases, or there may be some clinical significance other than infection. 


\subsection{The Possibility of a New Inflammatory Marker}

Another interesting result from this study was that observed elevations in blood endotoxin were not dependent on WBC count, CRP and inflammatory cytokine levels such as serum TNF- $\alpha$ or IL-6. Previous studies have pointed out that elevated endotoxin stimulates the production of inflammatory markers such as TNF- $\alpha$, IL- $1 \beta$, and IL-6 [24]. In this study, only endotoxin levels were significantly different between OP and SG groups while other inflammatory markers did not present such differences. Since no sign of infection other than endotoxin was seen in these measurements, the possibility has been suggested that blood endotoxin assay would be useful as an inflammatory marker independent of other factors. The study revealed no relationship between endotoxin levels and postoperative complications, since there were a limited number of cases, no sigh of postoperative infections and complications were present in any of the cases, and endotoxin levels did not exceed 0.6. However, there have been numerous reports in the past which point out a possible relationship between the two. In addition, the EAA method used in this study is more simplified and requires less time compared to the conventional method described above. Furthermore, since only EAA showed differences between open surgery and endovascular treatment on post-operatively period, it may be useful as a sensitive bio-marker to detect inflammation for abdominal aortic surgery. However, even in the case which exceeded baseline value (0.4) did not show any sign of infarction or other complication, further evaluation is necessary to set a cut-off value.

\section{Conclusion}

This study indicated that endovascular aneurysm repair was less invasive compared to open surgical repair from the aspect of perioperative EA levels. Furthermore, whole blood endotoxin activity levels measured using the EAA method were significantly elevated having no association with other inflammatory markers. This suggests that measurement of endotoxin activity by EAA method may become useful adjuncts to the clinician for the management of postoperative inflammation.

\section{Acknowledgements}

We thank Ms Yuko Kojima (Juntendo University, Graduate school) for her correcting English in this manuscript.

\section{References}

[1] Danner, R.L., Elin, R.J., Hosseini, J.M., et al. (1991) Endotoxemia in Human Septic Shock. Chest, 99, 169-175. http://dx.doi.org/10.1378/chest.99.1.169

[2] Jansen, P.G., TeVelthuis, H., Oudemans-Van Straaten, H.M., et al. (1994) Perfusion-Related Factors of Endotoxin Release during Cardiopulmonary Bypass. European Journal Cardio-Thoracic Surgery, 8, 125-129. http://dx.doi.org/10.1016/1010-7940(94)90167-8

[3] Klein, D.J., Briet, F., Nisenbaum, R., et al. (2011) Endotoxemia Related to Cardiopulmonary Bypass Is Associated with Increased Risk of Infection after Cardiac Surgery: A Prospective Observational Study. Critical Care, 15, R69. http://dx.doi.org/10.1186/cc10051

[4] Tsunooka, N., Hamada, Y., Imagawa, H., et al. (2003) Ischemia of the Intestinalmucosa during Cardiopulmonary Bypass. Journal of Artificial Organs, 6, 149-151.

[5] Boelke, E., Storck, M., Buttenschoen, K., et al. (2000) Endotoxemia and Mediator Release during Cardiac Surgery. Angiology, 51, 743-749. http://dx.doi.org/10.1177/000331970005100906

[6] Valenza, F., Fagnani, L., Coppola, S., et al. (2009) Prevalence of Endotoxemia after Surgery and Its Association with ICU Length of Stay. Critical Care, 13, R102. http://dx.doi.org/10.1186/cc7934

[7] Greenhalgh, R.M., Brown, L.C., Kwong, G.P., et al. (2004) Comparison of Endovascular Aneurysm Repair with Open Repair in Patients with Abdominal Aortic Aneurysm (EVAR Trial 1), 30-Day Operative Mortality Results: Randomised controlled Trial. Lancet, 364, 843-848. http://dx.doi.org/10.1016/S0140-6736(04)16979-1

[8] United Kingdom EVAR Trial Investigators, Greenhalgh, R.M., Brown, L.C., Powell, J.T., et al. (2010) Endovascular versus Open Repair of Abdominal Aortic Aneurysm. The New England Journal of Medicine, 362, 1863-1871. http://dx.doi.org/10.1056/NEJMoa0909305

[9] Lederle, F.A., Freischlag, J.A., Kyriakides, T.C., et al. (2012) Long-Term Comparison of Endovascular and Open Repair of Abdominal Aortic Aneurysm. The New England Journal of Medicine, 367, 1988-1997. http://dx.doi.org/10.1056/NEJMoa1207481 
[10] Jackson, R.S., Chang, D.C. and Freischlag, J.A. (2012) Comparison of Long-Term Survival after Open vs Endovascular Repair of Intact Abdominal Aortic Aneurysm among Medicare Beneficiaries. The Journal of the American Medical Association, 307, 1621-1628. http://dx.doi.org/10.1001/jama.2012.453

[11] Romaschin, A.D., Harris, D.M., Ribeiro, M.B., et al. (1998) A Rapid Assay of Endotoxin in Whole Blood Using Autologous Neutrophil Dependent Chemiluminescence. Journal of Immunological Methods, 212, 169-185. http://dx.doi.org/10.1016/S0022-1759(98)00003-9

[12] Marshall, J.C., Foster, D., Vincent, J.L., et al. (2004) Diagnostic and Prognostic Implications of Endotoxemia in Critical Illness: Results of the MEDIC Study. The Journal of Infectious Diseases, 190, 527-534. http://dx.doi.org/10.1086/422254

[13] Marshall, J.C., Walker, P.M., Foster, D.M., et al. (2002) Measurement of Endotoxin Activity in Critically Ill Patients Using Whole Blood Neutrophil Dependent Chemiluminescence. Critical Care, 6, 342-348. http://dx.doi.org/10.1186/cc1522

[14] Pathan, N., Burmester, M., Adamovic, T., et al. (2011) Intestinal Injury and Endotoxemia in Children Undergoing Surgery for Congenital Heart Disease. American Journal of Respiratory and Critical Care Medicine, 184, 1261-1269. http://dx.doi.org/10.1164/rccm.201104-07150C

[15] Soong, C.V., Blair, P.H.B., Halliday, M.I., et al. (1993) Endotoxaemia, the Generation of the Cytokines and Their Relationship to Intramucosal Acidosis of the Sigmoid Colon in Elective Abdominal Aorticaneurysm Repair. European Journal of Vascular Surgery, 7, 534-539. http://dx.doi.org/10.1016/S0950-821X(05)80366-4

[16] Fiddian-Green, R.G., Gantz, N.M. (1987) Transient Episodes of Sigmoid Ischemia and Their Relationshipto Infection from Intestinal Organisms after Abdominal Aortic Operations. Critical Care Medicine, 15, 835-839. http://dx.doi.org/10.1097/00003246-198709000-00007

[17] Bjorck, M. and Hedberg, B. (1994) Early Detection of Major Complications after Abdominal Aortic Surgery: Predictive Value of Sigmoid Colon and Gastric Intramucosal pH Monitoring. British Journal of Surgery, 81, 25-30. http://dx.doi.org/10.1002/bjs.1800810108

[18] Elmarasy, N.M., Soong, C.V., Walker, S.R., et al. (2000) Sigmoid Ischemia and the Inflammatory Response Following Endovascular Abdominal Aortic Aneurysm Repair. Journal of Endovascular Therapy; 7, 21-30. http://dx.doi.org/10.1583/1545-1550(2000)007<0021:SIATIR>2.3.CO;2

[19] Syk, I., Brunkwall, J., Ivancev, K., et al. (1998) Postoperative Fever, Bowel Ischemia and Cytokine Response to Abdominal Aortic Aneurysm Repair-A Comparison between Endovascular and Open Surgery. European Journal of Vascular and Endovascular Surgery, 15, 398-405. http://dx.doi.org/10.1016/S1078-5884(98)80200-1

[20] Thompson, M.M., Nasim, A., Sayers, R.D., et al. (1996) Oxygen Free Radical and Cytokine Generation during Endovascular and Conventional Aneurysm Repair. European Journal of Vascular and Endovascular Surgery, 12, 70-75. http://dx.doi.org/10.1016/S1078-5884(96)80278-4

[21] Norgren, L. and Swartbol, P. (1997) Biological Responses to Endovascular Treatment of Abdominal Aortic Aneurysms. Journal of Endovascular Surgery, 4, 169-173. http://dx.doi.org/10.1583/1074-6218(1997)004<0169:BRTETO>2.0.CO;2

[22] Swartbol, P., Norgren, L., Albrechtsson, U., et al. (1996) Biological Responses Differ Considerably between Endovascular and Conventional Aortic Aneurysm Surgery. European Journal of Vascular and Endovascular Surgery, 12, 18-25. http://dx.doi.org/10.1016/S1078-5884(96)80270-X

[23] Rocke, D.A., Gaffin, S.L., Wells, M.T., et al. (1987) Endotoxemia Associated with Cardiopulmonary Bypass. The Journal of Thoracic and Cardiovascular Surgery, 93, 832-837.

[24] Casey, L.C., Balk, R.A. and Bone, R.C. (1993) Plasma Cytokine and Endotoxin Levels Correlate with Survival in Patients with the Sepsis Syndrome. Annals of Internal Medicine, 119, 771-778. http://dx.doi.org/10.7326/0003-4819-119-8-199310150-00001 